Operationsgebiets sein, beispielsweise aufgrund der veränderten Morphologie oder bei Patienten mit Patella baja [13].

Keine der verfügbaren Studien wies radiologisch die besonders bei Implantation von achsgeführten Prothesen befürchtete erhöhte Lockerungstendenz nach $[12,13,14]$. Insgesamt scheint die Revisionsrate allerdings höher zu liegen als in der Allgemeinbevölkerung. Dies ist jedoch weniger mit dem Auftreten von Prothesenlockerungen, sondern vielmehr mit der muskuloligamentären Laxizität oder der erhöhten Sturzneigung vergesellschaftet.

\section{Fazit}

Sowohl bei Patienten mit Morbus Parkinson als auch bei solchen mit Poliomyelitis kann die Implantation einer KnieTEP zu einer deutlichen Reduktion der degerativ bedingten Knieschmerzen führen. Eine Verbesserung der Funktion ist jedoch nicht immer zur erwarten und vom Schweregrad der Grunderkrankung und dem Ausmaß der Weichteilund Knochenveränderungen abhängig. Aus diesem Grund spielt in beiden Patientengruppen postoperativ eine intensive krankengymnastische Beübung eine entscheidende Rolle. Bei Parkinsonpatienten wirkt sich zusätzlich eine frühzeitige neurologische Mitbehandlung günstig aus. Die Wahl des Prothesendesigns muss in beiden Patientengruppen individuell unter Berücksichtigung der ossären und muskulären Einschränkungen getroffen werden. Achsgeführte Prothesen erscheinen jedoch bei Patienten mit Poliomyelitis in vielen Fällen vorteilhaft. Bei Patienten mit Morbus Parkinson können posterior stabilisierte (PS)-Prothesen erfolgreich eingesetzt werden.

\section{Literatur bei den Verfassern}

Univ.-Prof. Dr.

Susanne Fuchs-Winkelmann

Dr. med. Dominik Malcherczyk

Klinik für Orthopädie und Rheumatologie

Baldinger Straße

35043 Marburg

E-Mail: orthopae@med.uni-marburg.de

\section{Operation Hüfte}

Patienten mit Hüftgelenkbeschwerden wissen oft nicht, wie sie zu einer angemessenen Bewertung ihres Leidens kommen, welche Behandlungsmöglichkeiten es gibt und wie eine Hüftgelenkersatzoperation abläuft. Für Orthopäden, die ihren hüftkranken Patienten - neben den ausführlichen persönlichen Informations- und Aufklärungsgesprächen - noch ein sinnvolle Lektüre zum Lesen daheim empfehlen wollen, ist die im Springer Verlag erschienene Broschüre "Operation Hüfte" eine gute Wahl.

Autor Robert Kipping beantwortet Fragen aus der Praxis rund ums Thema Hüftgelenk und gibt einen verständlichen Einblick in medizinisches Hintergrundwissen und Hüftprothesenmodelle. Er informiert über sinnvolle Selbstzahlerleistungen, die Beantragung einer Rehabilitation und die Möglichkeiten und Grenzen sozialmedizinischer Vergünstigungen.Der Autor ist seit knapp 25 Jahren im Bereich der operativen Orthopädie tätig und hat bislang weit über 7.000 Kunstgelenke implantiert.

Dr. Brigitta Schneider

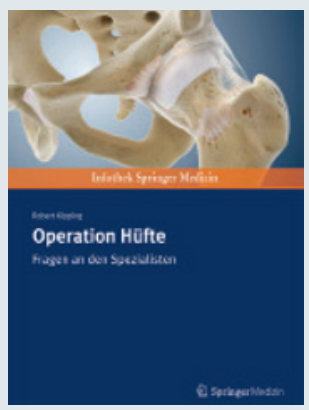

\section{Operation Hüfte}

Fragen an den Spezialisten

Kipping R.; Springer Verlag 2012; 88 S., 30 Abb.;

Brosch.; Ladenpreis (Preis inkl. MwSt.) 16,95 Euro

ISBN 978-3-89935-272-6

\title{
Gehen verstehen
}

— "So geht's" - mit dieser Überschrift beginnt die Autorin das zentrale Kapitel Ihres Buches über die Physiologie des menschlichen Gangbildes. Eine Sekunde nur dauert ein Gangzyklus und doch ereignet sich in dieser kurzen Zeit enorm viel: Zu bewältigen sind acht Gangphasen, kombiniert mit der permanenten Balance des Oberkörpers und weiteren differenzierten muskulären Aktivitäten.

Das vorliegende Buch beschreibt die exakte Vorgehensweise der beobachtenden Ganganalyse in der täglichen physiotherapeutischen Praxis. Die profunde Analyse dieses komplexen Geschehens wird ergänzt durch zahlreiche praktische Tipps und Hilfen zur Erstellung von Behandlungsansätzen und zur Schulung des Auges. Die Behandlung ausgewählter Störungen von der Beinachsenabweichung bis hin zur Spastik wird instruktiv und mit eindrucksvollen Abbildungen geschildert.

Frau Dr. J. Perry schreibt in ihrem Vorwort: „Wenngleich dieses Buch für Physiotherapeuten gedacht ist, bin ich überzeugt, dass es ebenso allen Klinikern und Wissenschaftlern willkommen ist, ,die nach umfangreichem Fachwissen über das Gehen verlangen." Das gilt selbstverständlich auch für jeden Orthopäden.

Prof. Klaus A. Milachowski

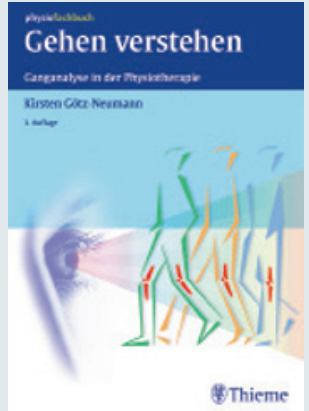

Gehen verstehen - Ganganalyse in der Physiotherapie. Kirsten Götz-Neumann; Thieme Verlag; geb.; 260 S..; 49,99 Euro (inkl. Mwst.); ISBN978-3-13-132373-6 